\title{
Study on Intestinal Parasitic Infections: Emphasis on Protozoan Parasites Entamoeba histolytica and Giardia lamblia and Associated Risk Factors Among School Children in Some Selected Primary Schools of Dembi Dolo Town, Western Ethiopia
}

\author{
Alemayehu Abdisa ${ }^{1} \quad$ Mebrate Dufera ${ }^{2 *}$ \\ 1.Kelem Secondary School, Dembi Dolo, Ethiopia \\ 2.Department of Biology, College Natural and Computational Sciences, Wollega University, Post Box No: 395 , \\ Nekemte, Ethiopia
}

\begin{abstract}
Intestinal parasitic infections caused mainly by protozoa and are most prevalent in tropical and subtropical regions of the world where adequate water and proper sanitation are lacking. Children are greatly prone to these infections via the ingestion of food, water or soil contaminated with the infective stage of these parasites. The aim of this study is to assess the status of intestinal parasitic infections giving emphasis to protozoan parasites Entamoeba histolytica and Giardia lamblia and Associated Risk Factors among primary school children of Dembi Dolo town. The cross sectional study was conducted in Grade 1 to 4 children in some selected Government and private schools of Dembi Dolo town. Stool samples were collected in May 2019, from 384 children, both male and female of equal proportion. The specimens were processed and then examined microscopically to determine the presence of intestinal parasites. Multivariate logistic regression was used to predict association between infection rates with age, sex and school type. The quantitative data was analyzed using SPSS version 20. The difference was considered statistically significant at $\mathrm{p}$-value $=0.05$. The intestinal parasitic infection rate for both protozoa and helminthes was 34.4\%. Overall prevalence of intestinal protozoan infections among the school children was $18 \%$. Entamoeba histolytica infection was higher (13.3\%) than Giardia lamblia $(4.7 \%)$. The prevalence of protozoan infection was slightly higher in males $(9.90 \%)$ than females $(8.07 \%)$, but the difference was not significant. The protozoan infection was higher among children whose age is lower compared to the higher age groups. The parasitic infection rates were significantly higher in Berhana Yesus school (38.3\%) compared to Burka Hayota (31.2\%). Majority of the children $97.4 \%$ had single species infections while $2.6 \%$ had multiple protozoa and helminthes infections. The personal hygienic factors associated with intestinal parasitic infections were failure to boil drinking water and wash hands with soap before eating. In conclusion there is relatively high prevalence of intestinal protozoan infections among the studied children. Therfore, on personal hygiene and environmental sanitation health education should be adopted in primary schools in order to reduce intestinal parasitic infections among school children.
\end{abstract}

Keywords: Intestinal parasites, Prevalence, Risk factors, School children

DOI: $10.7176 / \mathrm{JBAH} / 10-11-02$

Publication date:June $30^{\text {th }} 2020$

\section{Introduction}

Parasitic diseases continue to have a significant impact on the world's populations, especially in the lesserdeveloped regions of the world where delivery of health care, sanitation, and vector control efforts are less than adequate. However, the increased mobility of populations, immigration and development of populations due to civil strife are contributing factors that may extend their geographic range, or at the very least, create new public health concerns in previously unaffected areas (non-endemic areas). Both urbanization as well as movement in to suburban areas produces problems unique to both. Overcrowding in urban areas may test the adequacy of sanitation and control of the most common parasite species (WHO, 2011). Parasitic infections, and in particular those caused by protozoa, are a major public health problem. Intestinal parasitic infections are the most common infection worldwide. They are among the most widespread human infections in developing countries, with children being the most vulnerable population (Harhay, 2010). Parasites affect billion of people of all races and demographic regions. Intestinal parasitic infections are among the most common infections worldwide (Ukpai and Ugwu, 2003). Parasitic diseases represent a major cause of morbidity in most parts of the world (El -Masry et al., 2007).

The human is a habitat of 399 species of parasites (Ashford and Crewe, 1998). Of these, 197 species are reported to live in the alimentary tract (Crompton, 1999). The prevalence of intestinal parasites is the highest among children that are living in the poorest communities in developing nations. The most common causes of intestinal parasites are through consumption of contaminated water, infected soil, inadequate sanitation and hygiene, and improper hygiene (Ziegelbauer, 2012). Protozoa are microscopic, one-celled organisms that can be free-living or parasitic in nature. They are able to multiply in humans, which contributes for their survival and also permits serious infections to develop from just a single organism. The human intestinal protozoa include nonpathogenic and pathogenic parasites (WHO, 2014). Based on the world health organization (WHO) reports, 
approximately two-thirds of the world populations are infected with a wide range of parasitic protozoa. About 3.5 billion persons are infected with intestinal parasites and nearly 450 million suffer from clinical morbidity (WHO 2000). These reports also showed that, nearly 16 million of the total deaths occurring in developing countries are related to parasitic infections. According to earlier studies, children from developing countries, especially school age children, have the maximum rate of morbidity to intestinal parasites in comparison with other ages (Stephenson et al., 2000).

The protozoan parasites are the more common cause of gastrointestinal disorders compared to helminthes especially in developing countries. A number of intestinal protozoan parasites are reported in different parts of the world. Among them Entamoeba histolytica and Giardia lamblia are the major protozoan parasites of global health concern. Protozoan parasites being single celled can rapidly multiply inside the body leading to the development of the serious infection. Most of the protozoan infections tend to be asymptomatic. However, the common symptoms associated with it include abdominal discomfort, vomiting and dysentery. When burden of infection is pronounced, it may cause several complications like diarrhea, malaise, bloating, fatigue, epigastric discomfort, malnutrition, mal-absorption, intestinal ulceration, gastroenteritis, weight loss, abscesses, mental retardation, and even death. Protozoan infections can also lead to structural and functional abnormalities of small intestines in humans and can be misdiagnosed as appendicitis or other inflammatory diseases of gastrointestinal tract. Children are the primary victims of gastrointestinal protozoan parasites. So the disease control interventions need to be focused towards the pediatric group (Stephenson et al., 2000).

Poor access to safe water supply and hygiene are important risk factors for transmission of intestinal parasites. Primary school children frequently contact soil after and during school thus increasing chances of getting infected through ingestion of helminthes eggs and / or protozoa cysts due to poor personal hygiene. The children also involved in handling of food in homes hence if proper hygiene is not practiced they are likely to transmit infections to other people. Therefore, the study was initiated due lack of previous study in the area and inadequate water supply, poor hygiene among entire populations.

\section{Materials and Methods}

Study Area and period

The study was carried out in three primary schools within Dembi Dolo town of Western Ethiopia (Figure 1). Dembi Dolo is the capital of KelemWelega Zone of the Oromia Region. The town has a latitude and longitude of $8^{0} 32 \mathrm{~N}$ $3448 \mathrm{E} / 8.533^{\circ} \mathrm{N} 34.800^{\circ} \mathrm{E}$ with an elevation between 1701 and 1827 meters above sea level. The town is characterized by moist midland, the annual rainfall $1670.65 \mathrm{~mm}$ and the mean annual temperature is $19.45 \mathrm{oC}$ (Dembi Dolo town communication office). The town is bounded at four sides by Seyo Wereda. The 2007 national census reported a total population for this town of 29,448, of whom 15,144 were men and 14,304 were women. The majority of the inhabitants were Protestants, with 58.23\% reporting that as their religion, while $30.14 \%$ observed Ethiopian Orthodox Christianity, 8.81\% observed Islam, and 2.07 were catholic. The study was conducted from February 10 to August 30/2019.

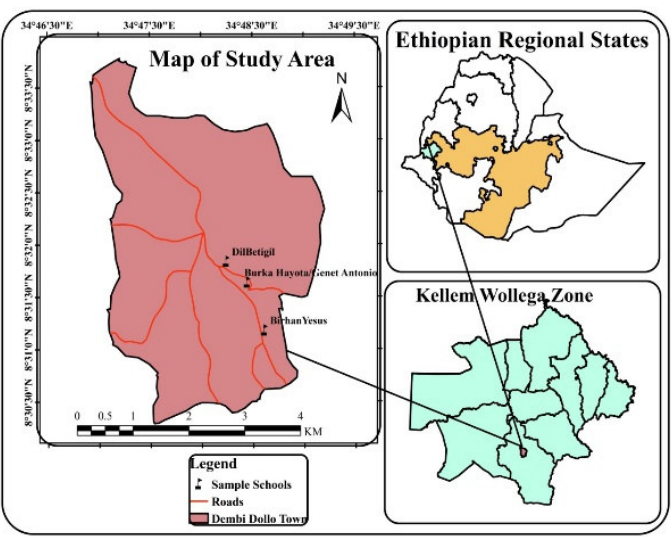

Figure I: Map of Study area (Source: Garmin 72 GPS)

\section{Study Population}

The study population were all school children whose age were 7-13 years who were attending their education at public schools and the sample population were the selected school children from the selected schools(Burka Hayota, Berhana Yesus and Dil Betigil primary schools) in Dembi Dollo town of western Ethiopia.

\section{Study Design}

This was a cross sectional descriptive study involving some selected primary school children in Dembi Dolo town. 


\section{Sample Size Determination and Sampling Technique}

The sample size in this study was calculated by using single proportion formula at $95 \%$ confidence interval level $(\mathrm{Z}(1-\alpha / 2)=1.96)$ and $5 \%$ marginal error. Then, the sample size were calculated by using the formula $n=[\mathrm{Z} 1-\mathrm{a} / 2] 2$ $\mathrm{p}(1-\mathrm{p}) / \mathrm{d} 2=384$.

The number of children sampled in each class was then proportionately distributed among each of the four classes. In the class 35 children were selected from an average of 50 children per class. The selection was done from children present in class using the class register by allocating random numbers.

\section{Stool Collection and Laboratory Examination Procedures}

A total of 422 stool specimens were collected from both male and female of one, two and three children from the three primary schools. The children were given sterile, capped specimen bottles labeled with random numbers, and an applicator stick. They were instructed to collect a first morning stool specimen. Stool specimen for each of the classes were collected on three consecutive days per week beginning from Grade one to Grade Four. The stool specimens from the school were examined usining Direct wet mounts to observe the presence or absence of protozoa cysts and / or trophozoites according to laboratory practice (Cheesebrough, 2001) at Dambi Dolo Hospital by two qualified laboratory technicians. Randomly picked stool specimen were re-examined by a third technician to increase the accuracy of results.

\section{Data Collection}

Data on the occurrence of intestinal parasitic infections among children was obtained by laboratory investigations of the collected stool specimen. Data on personal hygiene, social demographic and environmental factors was collected using predesigned questionnaires. The questionnaires were prepared in two languages (English and Afan Oromo). The questionnaires was filled by children one day before collection of stool specimen after which they are given specimen sterile specimen bottles to bring stool specimen the following morning. A check list on the environmental sanitation in school was filled by boarding masters, who are teachers in the respective schools. The stool specimens were collected as described below. The questionnaires contained three sections; the first section was on social demographic factors such as age, class, residence, the second section was on environmental factors including water source, type of toilets, presence of flies in toilet and / or latrines, and the last section was on hygiene practices such as washing hands, fruits and other food items. Some samples of the questionnaires were pretested on two children randomly selected from each of the four classes of the school.

\section{Data Analysis}

The quantitative data was coded and entered into a computer data base designed using SPSS version 20 and data cleaning and validation were performed in order to achieve a clean dataset by the software. The rates of intestinal parasitic infections among the school children based on their sex and age was analyzed by expressing the positive samples as a percentage of the total number of children sampled. The data was compared using multi variant regression and Analysis of Variance (ANOVA) to predict whether there is significant association. Adjusted odds ratio (AOR) was used to determine the association of environmental and personal hygiene practices and intestinal parasitic infections. Binary logistic regression was conducted at $\mathrm{p}<0.2$ and the potential variables were entered in to multivariable logistic regression. The difference was considered significant at a $p<0.05$. Quantitative data was presented using tables and graphs.

\section{Treatment}

Children' participation in the study was fully voluntary and confidentiality was maintained during the study. The procedure was non-invasive hence not harmful to the subjects. Positive cases for any intestinal parasite were treated according to FMHACA, Standard Treatment Guidelines for General Hospital, $3^{\text {rd }}$ Edition, 2014.

\section{Ethical clearance/considerations}

The study was approved by the graduate school, Wollega University. The ethical clearance was obtained from the Research Ethics Review Committee (RERC) of Wollega University. Written informed consent was obtained from each guardian for individuals below 18 years old. Further, no loss or harm came to the study participants of this study. In addition confidentiality of data and information from this study was maintained.

\section{Results \\ Socio demographic factors}

Out of 384 children, 192(50\%) were males and 192(50\%) females. Majority, 131(34.1\%) were 7-8 years old followed by $129(33.6 \%)$ and $124(32.3 \%)$ in the age groups 9-10 and 11-13 respectively. Concerning their residence, 319(83.1\%) were from Dembi Dolo town and 65(16.9\%) from surrounding rural areas (Table 1) 
Table 1: Socio-demographic data and rate of infection with intestinal parasite $(n=384)$

\begin{tabular}{cccccccccc}
\hline \multicolumn{1}{c}{ Sex } & \multicolumn{4}{c}{ Grade level } & \multicolumn{3}{c}{ Age in year } & Infection \\
rate, $\mathrm{n}(\%)$
\end{tabular}

\section{Occurrence of Intestinal Infections among Children}

Out of the total 422 children recruited at the start of the study, only 384 among them provided stool specimen for three consecutive weeks. They also completed all the information required on the questionnaires. Of these children, $50 \%$ were males and another 50\% were females. The intestinal parasitic infection rate for both protozoa and helminthes was $34.4 \%$. The infections due to protozoa were $18 \%$ and $16.4 \%$ were due to helminthes, while multiple infections were $2.6 \%$.

The types of intestinal parasitic infections found in the sampled children were Entamoeba histolytica/dispar, Giardia lamblia, Ascaris lumbricoides, Hymenolepsis nana and hookworms. Among the protozoan infections Entamoeba histolytica/dispar had the highest infection rate (13.3\%). Ascaris lumbricoides had the highest infection rate $(9.90 \%)$ among the helminthes (Figure 2).

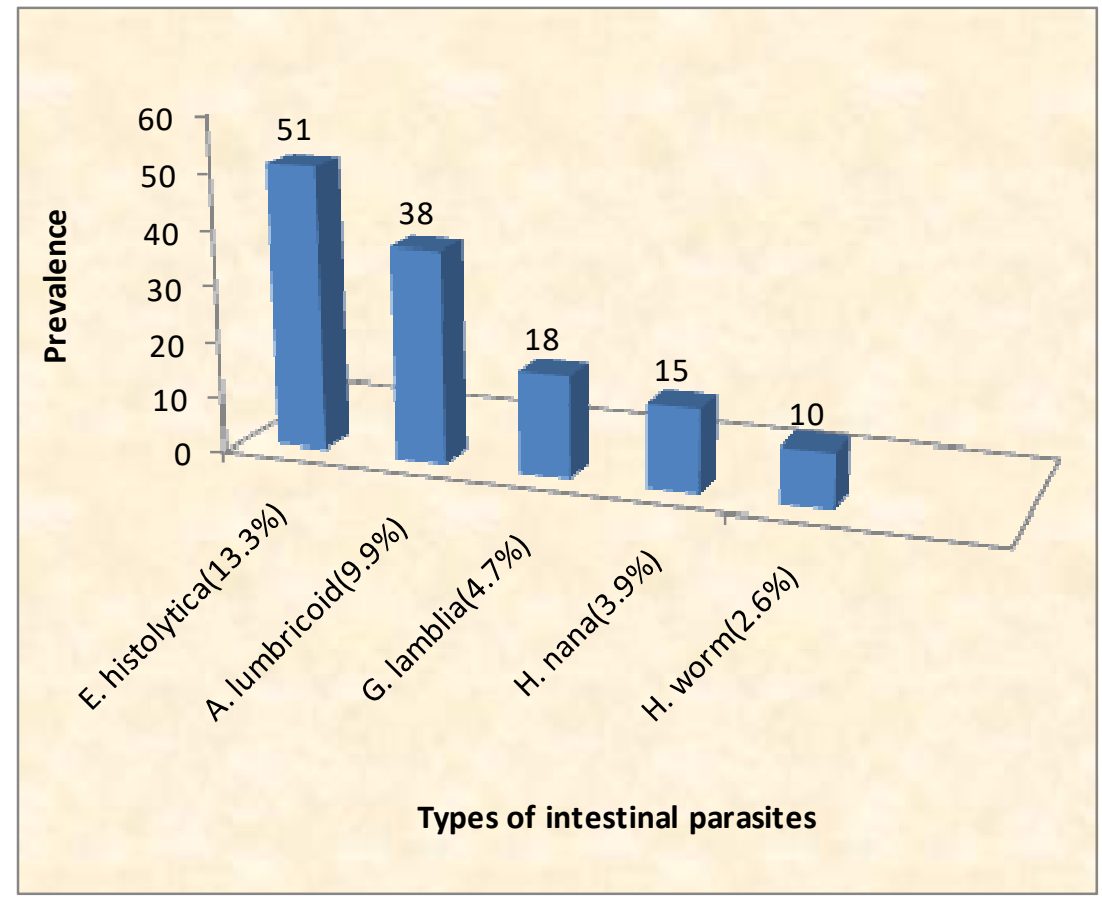

Figure 2: Infection rates of Intestinal Parasites among the studied children

There were variations in intestinal parasitic infections with respect to age in the studied children. Children of 7-8 years age group recorded highest prevalence of pathogenic intestinal parasites whiles children within the age group 11-13 years recorded the lowest. Among the pathogenic intestinal parasites, it was observed that prevalence decreases with advancement in age (Figure 3). 


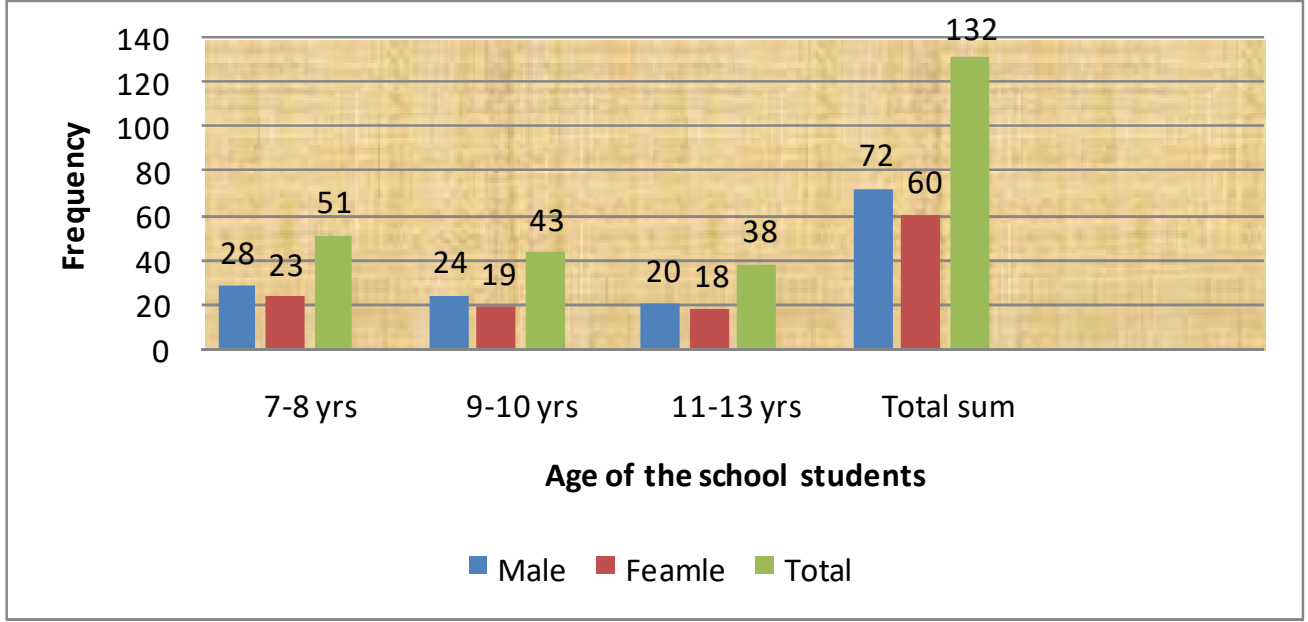

Figure3: Age distribution of Intestinal Parasites among the studied children

Five intestinal parasites were identified in the study: two protozoans and three helminthes species. The commonest intestinal parasites associated with the children were Entamoeba histolytica, followed by Ascaris lumbricoid while hook worm was the least. The most common protozoa in all the schools, Entamoeba histolytica/dispar was (13.3\%), and there was significant difference in the infection rates due to protozoa infections among the different schools (Table 3). Ascaris lumbricoides infections were the most common among the helminthes in all the schools sampled at $9.90 \%$ while Hook worm was the least at $2.6 \%$ (Table 2).

Table 2: Infection rates of various species of intestinal parasites per school

\begin{tabular}{|c|c|c|c|c|c|c|c|}
\hline \multirow[b]{2}{*}{ Parasites } & \multicolumn{3}{|c|}{ Schools } & \multirow{2}{*}{$\begin{array}{l}\text { Total } \\
\text { infections } \\
\mathrm{n}=384 ; \\
\mathrm{n}(\%)\end{array}$} & \multirow{2}{*}{ COR } & \multirow{2}{*}{ CI } & \multirow[b]{2}{*}{$\begin{array}{l}\mathrm{p}- \\
\text { value }\end{array}$} \\
\hline & $\begin{array}{l}\text { Burka } \\
\text { Hayota } \\
\text { n=128; } \\
\text { n(\%) }\end{array}$ & $\begin{array}{c}\text { BerhanaYesus } \\
\text { n=128; } \\
n(\%)\end{array}$ & $\begin{array}{c}\text { Dil Betigil } \\
\text { n=128; } \\
n(\%)\end{array}$ & & & & \\
\hline E.histolytica & $15(11.72 \%)$ & $18(14.1 \%)$ & $18(14.1 \%)$ & $51(13.3 \%)$ & 1.02 & $1.15-1.25$ & 0.00 \\
\hline G.lamblia & $7(5.5 \%)$ & $5(3.91 \%)$ & $6(4.7 \%)$ & $18(4.7 \%)$ & 2.05 & $1.02-3.10$ & 0.005 \\
\hline Total protozoa & $22(17.2 \%)$ & $23(17.97 \%)$ & $24(18.75 \%)$ & $69(18 \%)$ & 2.01 & $1.91-2.03$ & 0.00 \\
\hline A. lumbricoids & $10(7.81 \%)$ & $16(12.5 \%)$ & $12(9.4 \%)$ & $38(9.9 \%)$ & 1.09 & $1.11-1.53$ & 0.02 \\
\hline Hook worm & $3(2.34 \%)$ & $4(3.12 \%)$ & $3(2.34 \%)$ & $10(2.6 \%)$ & 2.70 & $1.03-5.09$ & 0.705 \\
\hline H.nana & $5(3.91 \%)$ & $6(4.7 \%)$ & $4(3.12 \%)$ & $15(3.91 \%)$ & 3.01 & $1.00-4.04$ & 0.004 \\
\hline $\begin{array}{l}\text { Total } \\
\text { helminthes }\end{array}$ & $18(14.1 \%)$ & $26(20.31 \%)$ & $19(14.84 \%)$ & $63(16.41 \%)$ & 1.92 & $0.81-2.35$ & 0.003 \\
\hline Total parasites & $40(31.25 \%)$ & $49(38.28 \%)$ & $43(33.59 \%)$ & $132(34.4 \%)$ & 1.50 & $0.99-2.0$ & 0.01 \\
\hline
\end{tabular}

Comparing infection rates by intestinal protozoa and helminthes among different groups, it was shown that infection rates were highest among group one (7-8years of age) children (38.93\%) followed by group two (9-10 years of age) children (33.33\%) while group three (11-13 years of age) children had the least at (30.65\%) in all the three schools combined (Table 3 ). There were variations in intestinal protozoan infections with respect to age in the studied children. Children of 7-8 years age group recorded highest prevalence of pathogenic intestinal protozoan infection whiles children within the age group 11-13 years recorded the lowest. Hence, it was observed that prevalence decreases with advancement in age.

Table 3: Infection rates of protozoa and helminthes among different age groups

\begin{tabular}{|c|c|c|c|c|c|}
\hline \multirow[b]{2}{*}{ Group } & \multicolumn{3}{|c|}{ School } & \multirow{2}{*}{$\begin{array}{c}\text { Total } \\
\mathrm{n}=384 ; \\
\mathrm{n}(\%)\end{array}$} & \multirow[t]{2}{*}{ P-value } \\
\hline & $\begin{array}{l}\text { Burka Hayota } \\
\mathrm{n}=128 ; \quad \mathrm{n}(\%)\end{array}$ & $\begin{array}{c}\text { Berhana Yesus } \\
n=128 \\
n(\%)\end{array}$ & $\begin{array}{l}\text { Dil Betigil } \\
\text { n=128; } \\
\mathrm{n}(\%)\end{array}$ & & \\
\hline $\begin{array}{l}7-8 \text { yrs of age } n=131 \text { (group } \\
\text { 1) }\end{array}$ & $16(12.2 \%)$ & $20(15.3 \%)$ & $15(11.45 \%)$ & $51(38.93 \%)$ & $\mathrm{P}=0.001$ \\
\hline $\begin{array}{l}9-10 \text { yrs of age } \\
n=129(\text { group } 2)\end{array}$ & $14(10.85 \%)$ & $16(12.4 \%)$ & $13(10.1 \%)$ & $43(33.33 \%)$ & $\mathrm{P}=0.055$ \\
\hline $\begin{array}{l}11-13 \text { yrs of age } \\
n=124(\text { group } 3) \\
\text { Total }\end{array}$ & $\begin{array}{c}10(7.75 \%) \\
40(10.42 \%)\end{array}$ & $\begin{array}{c}13(10.1 \%) \\
49(12.76 \%)\end{array}$ & $\begin{array}{l}15(11.63 \%) \\
43(11.2 \%)\end{array}$ & $\begin{array}{l}38(30.65 \%) \\
132(34.4 \%)\end{array}$ & $\mathrm{P}=0.093$ \\
\hline
\end{tabular}


Out of 384 children, $192(50 \%)$ were males and 192(50\%) females. Majority, 131(34.1\%) were 7-8 years old followed by $129(33.6 \%)$ and $124(32.3 \%)$ in the age groups 9-10 and 11-13 respectively. Concerning their residence, $319(83.1 \%$ ) were from Dembi Dolo town and 65(16.9\%) from surrounding rural areas. To quantify the relationship between the infection rate, highest age, grade 4 and females was used as the reference test respectively. The statistical analyses demonstrated significant differences between microscopy and concentration method $(\mathrm{P}=0.04)$. Concentration method revealed by far the highest positivity rate $(9.4 \%)$ compared to the microscopic methods. To quantify the relationship between the techniques, microscopy was used as the reference test (Table 4).

Table 4: Multivariate logistic regression analysis of socio-demographic data in relation to intestinal protozoa/helminthes-positive, $(\mathrm{n}=384)$

\begin{tabular}{|c|c|c|c|c|c|c|}
\hline Variables & Alternatives & Number & + ve, n(\%) & AOR & $95 \% \mathrm{CI}$ & P-value \\
\hline \multirow{3}{*}{ Age } & $7-8$ & 131 & $51(38.9 \%)$ & 2.3 & $1.32-5.21$ & 0.001 \\
\hline & $9-10$ & 129 & $43(33.3 \%)$ & 1.9 & $1.12-2.50$ & 0.02 \\
\hline & $11-13$ & 124 & $38(30.6 \%)$ & 1 & 1 & 1 \\
\hline \multirow{3}{*}{ Sex } & M & 192 & $72(37.5 \%)$ & 2.03 & $1.88-4.31$ & 0.03 \\
\hline & $\mathrm{F}$ & 192 & $60(31.3 \%)$ & 1 & 1 & 1 \\
\hline & 1 & 96 & $39(40.6 \%)$ & 3.02 & $1.63-6.88$ & 0.02 \\
\hline Grade & 2 & 96 & $33(34.4 \%)$ & 0.63 & $0.03-1.06$ & 0.08 \\
\hline \multirow[t]{3}{*}{ Level } & 3 & 96 & $32(33.3 \%)$ & 0.35 & $0.01-1.65$ & 0.06 \\
\hline & 4 & 96 & $28(29.2 \%)$ & 1 & 1 & 1 \\
\hline & Urban & 319 & $111(28.5 \%)$ & 1 & 1 & 1 \\
\hline Residence & Rural & 65 & $21(5.7 \%)$ & 2.01 & -6.12 to -1.06 & 0.04 \\
\hline \multirow[t]{2}{*}{ Methods } & Microscopy & 320 & $104(27.1 \%)$ & 1 & 1 & 1 \\
\hline & Ether conc. & 64 & $28(7.3 \%)$ & 0.73 & $3.14-7.21$ & 0.03 \\
\hline
\end{tabular}

The male children had an overall infection rate of $37.50 \%$, which was slightly higher compared to females at $31.3 \%$ (Table 5). Infection rate with both protozoa and helminthes were slightly higher in males than in females at $9.90 \%$ and $8.85 \%$ respectively for protozoa, and $8.07 \%$ and $7.55 \%$ respectively for helminthes. Most scholars have attributed this skewness to socio-cultural and behavioural differences between males and females children. Males obtained higher prevalence of intestinal parasitic infection because they get more freedom than females whose leisure hours are strictly controlled and restricted hence are less exposed to parasitic infections.

Table 5: Intestinal Parasitic Infections by sex $(n=384)$

\begin{tabular}{llll}
\hline Intestinal parasites & $\begin{array}{l}\text { Males } \mathrm{n}=192 \\
\mathrm{n}(\%)\end{array}$ & $\begin{array}{l}\text { Females } \mathrm{n}=192 \\
\mathrm{n}(\%)\end{array}$ & $\begin{array}{l}\text { Total } \\
\mathrm{n}(\%)\end{array}$ \\
\hline E. histolytica & $27(14.1 \%)$ & $24(12.5 \%)$ & $51(13.3 \%)$ \\
G. lamblia & $11(5.7 \%)$ & $7(3.65 \%)$ & $18(4.7 \%)$ \\
Total protozoa & $38(19.8 \%)$ & $31(16.2 \%)$ & $69(18 \%)$ \\
A. lumbricoids & $21(10.9 \%)$ & $17(8.85 \%)$ & $38(9.9 \%)$ \\
Hook worms & $5(2.6 \%)$ & $5(2.6 \%)$ & $10(2.6 \%)$ \\
H. nana & $8(4.2 \%)$ & $7(3.65 \%)$ & $15(3.9 \%)$ \\
Total helminthes & $34(17.7 \%)$ & $29(15.1 \%)$ & $63(16.4 \%)$ \\
Total Intestinal parasites & $72(37.50 \%)$ & $60(31.25 \%)$ & $132(34.4 \%)$ \\
\hline
\end{tabular}

\section{Co-Infection of Protozoa and Helminthes Intestinal among Children}

Most of the infections among children were of single species. However, $2.6 \%$ of the children tested had multiple infections. The most common co-infections were recorded between A. lumbricoides and E. histolytica/dispar $(0.78 \%)$. Berhana Yesus primary school had significantly higher co-infection rate of $1.30 \%$ compared to Dil Betigil school $0.78 \%$ and Burka Hayota at $0.52 \%$. Co-infections of A. lumbricoides and H. nana were recorded only in Berhana Yesus school (Table 6).

Table 6: Co-infection rates of protozoa and helminthes among the three schools $(n=384)$

Intestinal parasites

A. lumbricoides and E. histolytica

A. lumbricoides and H.nana

E. histolytica and H.nana

Total co-infections
Sample schools

\begin{tabular}{llll}
\cline { 1 - 1 } $\begin{array}{l}\text { Burka Hayota } \\
\mathrm{n}(\%)\end{array}$ & $\begin{array}{l}\text { Berhana Yesus } \\
\mathrm{n}(\%)\end{array}$ & $\begin{array}{l}\text { Dil Betigil } \\
\mathrm{n}(\%)\end{array}$ & $\begin{array}{l}\text { Total } \\
\mathrm{n}(\%)\end{array}$ \\
\hline $1(0.26 \%)$ & $3(0.78 \%)$ & $2(0.52 \%)$ & $6(1.56 \%)$ \\
$0(0 \%)$ & $1(0.26 \%)$ & $0(0 \%)$ & $1(0.26 \%)$ \\
$1(0.26 \%)$ & $1(0.26 \%)$ & $1(0.26 \%)$ & $3(0.78 \%)$ \\
$2(0.52 \%)$ & $5(1.30 \%)$ & $3(0.78 \%)$ & $10(2.6 \%)$ \\
\hline
\end{tabular}


Intestinal parasites in relation to family background

The protozoan infection was higher among children whose parents were illiterates $(41.54 \%)$ than among primary $(45.0 \%)$ and secondary and above (28.0\%) schools completed individuals (Table 7). Increased risk of infection was found in children who were their parents involved in farming activities compared to those not involved in farming activities.

Table 7: Socio- demographic factors of intestinal parasites in relation to family background $(\mathrm{n}=384)$

\begin{tabular}{llcc}
\hline Characteristics & & $\begin{array}{c}\text { Number of children, } \\
\mathrm{n}(\%)\end{array}$ & $\begin{array}{c}\text { Infected children, } \\
\mathrm{n}(\%)\end{array}$ \\
\hline Family size & Three to five & $164(42.71 \%)$ & $49(29.88 \%)$ \\
Educational level & $220(57.29 \%)$ & $83(37.73 \%)$ \\
& More than five & $65(16.93 \%)$ & $27(41.54 \%)$ \\
& Illiterate & $173(45.05 \%)$ & $78(45.09 \%$ \\
Work & Read and write & $146(38.02 \%)$ & $41(28.08 \%)$ \\
& Secondary and above & $141(36.7 \%)$ & $45(31.9 \%)$ \\
& Employee & $79(20.6 \%)$ & $29(36.7 \%)$ \\
& Farmer & $54(14.1 \%)$ & $19(35.2 \%)$ \\
& Merchant & $110(28.6 \%)$ & $39(35.4 \%)$ \\
\hline
\end{tabular}

Transmission and Associated Risk factors in relation to Intestinal Parasitic Infections among Children Concerning the risk factors of intestinal parasitic infections in relation to environmental sanitation within the homes majority of them used unimproved water sources (71.61\%).Also, most of the children (78.65\%) used pit latrines in homes as opposed to flush toilets. The study showed that $57.29 \%$ were infested with flies and / or cockroaches (Table 8).

Table 8: Transmission and Associated Risk factors in relation to home environment $(\mathrm{n}=384)$

\begin{tabular}{lcc}
\hline Environmental factors & $\begin{array}{c}\text { Number of children } \\
\mathrm{n}(\%)\end{array}$ & $\begin{array}{c}\text { Infected children } \\
\mathrm{n}(\%)\end{array}$ \\
\hline Improved water sources & $109(28.4 \%)$ & $21(5.47 \%)$ \\
Unimproved water sources & $275(71.6 \%)$ & $111(28.9 \%)$ \\
Pit latrines & $302(78.6 \%)$ & $118(30.7 \%)$ \\
Flush latrines & $82(21.3 \%)$ & $14(17 \%)$ \\
Presence flies and/ or cockroaches in sanitary facilities & $220(57.3 \%)$ & $72(32.7 \%)$ \\
Absence of flies and /or cockroaches in sanitary facilities & $164(42.7 \%)$ & $60(36.5 \%)$ \\
\hline
\end{tabular}

All the three schools sampled obtained water from unimproved sources and did not have had hand washing facilities close to the latrines and dining facilities (Table 9). None of the three schools sampled provided children with soap for washing hand after visiting toilets or before meals. All the three schools sampled had pit latrines for the children. Children in all the schools cited seeing flies and cockroaches in the latrines and toilets. Table 9: Environmental factors that influence transmission of intestinal parasites within the schools

\begin{tabular}{|c|c|c|c|c|}
\hline \multirow[b]{2}{*}{ Risk factors } & \multirow[b]{2}{*}{ Conditions } & \multicolumn{3}{|c|}{ Schools } \\
\hline & & Burka Hayota & Berhana Yesus & Dil betigil \\
\hline \multirow[t]{2}{*}{ Water sources } & Improved & $x$ & $x$ & $x$ \\
\hline & Unimproved & $\sqrt{ }$ & $\sqrt{ }$ & $\sqrt{ }$ \\
\hline \multirow[t]{2}{*}{ Provision of safe drinking water } & Provide & $x$ & $x$ & $x$ \\
\hline & Don't provide & $\sqrt{ }$ & $\sqrt{ }$ & $\sqrt{ }$ \\
\hline \multirow[t]{2}{*}{ Provision of hand washing facilities } & Provide & $\times$ & $\times$ & $x$ \\
\hline & Don't provide & $\sqrt{ }$ & $\sqrt{ }$ & $\sqrt{ }$ \\
\hline \multirow[t]{2}{*}{ Provision of soap } & Provide & $\times$ & $\times$ & $x$ \\
\hline & Don't provide & $\sqrt{ }$ & $\sqrt{ }$ & $\sqrt{ }$ \\
\hline \multirow[t]{2}{*}{ Type of sanitary facilities } & Pit latrines & $\sqrt{ }$ & $\sqrt{ }$ & $\sqrt{ }$ \\
\hline & Flush toilets & $\times$ & $\times$ & $x$ \\
\hline \multirow[t]{2}{*}{ Flies and / or cockroaches in sanitary facilities } & Present & $\sqrt{ }$ & $\sqrt{ }$ & $\sqrt{ }$ \\
\hline & Absence & $x$ & $x$ & $x$ \\
\hline
\end{tabular}

Personal hygiene practices influences transmission of intestinal parasitic infections. The results showed that only $32.7 \%$ of the children boiled drinking water. Boiling drinking water was significantly associated with reduced likelihood of infection with intestinal parasites. A large number of children did not wash hand after visiting toilets or before meals. Of these, $66.06 \%$ were infected with intestinal parasites. The children who washed their hands with soap after visiting the toilets and before meals were less likely to be infected with intestinal parasites However washing hands with water only without soap did not significantly reduce infections with intestinal parasites. About $27.5 \%$ of the children washed fruits before eating. This significantly reduced their chance of infection with 
intestinal parasites .About $60 \%$ of the children eat food sold in open places, this practice was however not associated with infection with intestinal parasites (Table 10).

Table 10: Personal hygiene practices that influence transmission of intestinal parasites

\begin{tabular}{lll}
\hline Personal hygienic practices & $\begin{array}{l}\text { Number of } \\
\text { children }\end{array}$ & Children infected n(\%) \\
\hline Boiling drinking water & 58 & $13(22 \%)$ \\
Not boiling drinking water & 326 & $165(50.6 \%)$ \\
Washing hands after visiting toilets & 219 & $95(43 \%)$ \\
Not washing hands after visiting toilets & 165 & $109(66 \%)$ \\
Washing hands with soap & 130 & $44(33.8 \%)$ \\
Not washing hands with soap & 254 & $132(51.9 \%)$ \\
Washing fruits before eating & 203 & $56(27.5 \%)$ \\
Not washing fruits before eating & 181 & $83(45.8 \%)$ \\
Eating food sold in open places & 175 & $105(60 \%)$ \\
Not eating food sold in open places & 209 & $99(47 \%)$ \\
Sources of fruits \& vegetables from market & 186 & $69(37 \%)$ \\
Sources of fruits \& vegetables from farm & 198 & $107(54 \%)$ \\
\hline
\end{tabular}

\section{Discussions}

Most of the existing data on intestinal parasitic infections has been based on school age children of 14-20 years old. The current study accessed the occurrence of intestinal parasitic infections among school attending children aged between 7-13 years in three primary schools in Burka Hayota, Berhana Yesus and Dil Betigil in Dembi Dolo town. The overall infection rate for both protozoa and helminthes was $34.4 \%$.

The total prevalence of protozoa in this study was $18 \%$ and that of helminthes were $16.4 \%$. The types of intestinal parasitic infections found in the sampled children were protozoa; Entamoeba histolytica/dispar (13.3\%) and Giardia lamblia (4.7\%). In addition to trichuris trichiura the same intestinal parasites were identified by the research conducted in Addis Ababa (Mekonn et al., 2014).

In Ethiopia this result (13.3\%) was relatively higher when compared to the finding of Mojo health center, eastern Ethiopia (2\%) in the year 2009 (Bayissa 2013). On the other hand, the rate was relatively goes in line when compared to the findings of study conducted at Tikur Anbesa hospital showed that Entamoeba histolytica/dispar was the most common reported parasite with 13.6\% prevalence rate (Petri and Sing 1999) and was lower when compared with the study in semi-pastoralist tribes in lower Omo Valley, Southwestern Ethiopia by Teklehaymanot (2009) $(16 \%)$.

The rate of Giardia lamblia in this study (4.7\%) was relatively similar (4.3\%) regarding the result recorded in Awuramba, reported by Yihinew (2011), and 4.4\% in Anambra state reported by Emmy-Egbi et al. (2012), but it was relatively lower than $4.9 \%$ in Gorgora and chuahit health center North Gondar reported by Abate et al. (2013), 6.6\% in Teda Health center North Gondar, 6.7\% in Babile town Eastern Ethiopia reported by Tadesse (2005) and 6.4\% in Nigeria reported by Auta et al. (2013).

In this study, the prevalence of parasitic infection was seen slightly higher in males $(37.5 \%)$ than females $(31.3 \%)$ but the difference was not significant. Most scholars have attributed this skewness to socio-cultural and behavioural differences between males and females children.

A similar study conducted by Sah et al. (2013) in Dharan, Nepal which also showed higher infection rate of protozoa in males $(14.3 \%)$ than females (11.4\%). Other studies for example, done in Bondo District, Kenya among children aged between 5-20 years showed a higher infection rate in boys than in girls at 39.0\% compared to $34.5 \%$ ) (Thiongo, et al., 2009). Studies done in China indicated a higher infection rate 53\% among the male children compared to $39 \%$ among female children aged between 10-23 years (Wang et al., 2012). On the contrary a study conducted by Alyousefi et al.,(2011) in Sana'a city, Yemen showed that the infection rate of protozoa was lower in males $(29.4 \%)$ than females $(32.2 \%)$.

In this study slightly higher levels of infection (38.98\%) were recorded among Group one (7-8 years of age) student compared to those in Group two (9-10 years of age) and Group three (11-13 years of age) at 33.3\% and $30.65 \%$ respectively. This could be because group one children are younger hence may have been exposed to variety of contaminated environments. According to WHO (2010), infection rates vary by age groups in which prevalence is higher in lower age groups. This result goes in line with studies done in Western Kenya that indicated a higher infection rate among children aged below ten years $(47 \%)$ compared to those aged above ten years $(30 \%)$ (Thiong'o et al., 2009).

When compared relatively the infection rate of the three schools, Berhana Yesus primary school had the highest infection rate (37.5\%) compared to Dil Betigil (33.6\%) and Burka Hayota $(31.3 \%)$. This could be about $40 \%$ of the children of the Berhana Yesus primary school had come from villages. In the village, the source of water is stream which could be contaminated with faeces from wild animals. Additionally, the families are 
involved in farming activities and there is a lack of appropriate food hygiene.

Multiple infections of protozoa and helminthes existed in only $2.6 \%$ of the children sampled. The highest coinfection rate recorded in this study was between A. lumbricoides and E. histolytica/dispar at $23.1 \%$ and higher co-infection were recorded relatively in male children.

The study showed that all the three schools sampled had pit latrines as opposed to flush toilets. Construction of toilets and proper usage has been suggested as an effective control measure of intestinal parasites. In circumstances where toilets are not easily cleaned and the water table is high protozoan infections may not be effectively controlled. The study reported presence of flies and /or cockroaches in pit latrine both at home and in school, indicating that they were not well maintained hygienically. These vectors potentially carry the protozoan cysts mechanically and deposited them on food leading to infections. They could also be a source of infection for the latrine users especially if they do not wash hands after latrine use.

The study indicates that $54.7 \%$ of children used unimproved water sources in their homes. These children were found to have higher protozoa infections than those using improved water sources. In addition all the schools sampled did not provide safe drinking water for the children. These factors could have lead to increase in transmission of intestinal parasites especially Entamoeba histolytica/dispar and Giardia lamblia that are commonly transmitted through fecal contamination of drinking water.The discrepancies between the present and other previous studies may be due to differences between studied population characteristics or geographical and socioeconomic factors, and prevailing climatic and social conditions in the areas of study.

\title{
Conclusions
}

The study revealed that the present prevalence of intestinal protozoan infection among primary school children aged 7 to 13 years in the Dembi Dolo town was 34.4\%. Entamoeba histolytica/dispar was the commonest pathogenic protozoan in the studied children followed by Giardia lamblia recorded the second of protozoan parasite. The total occurrence of protozoa was $18 \%$ and helminthes infection rates were $16.4 \%$. The most common protozoan infection was due to E. histolytica/dispar at $13.3 \%$ while $G$. lamblia infections was $4.7 \%$. Most of the infections among children were single protozoa and helminthes species infections at $97.4 \%$ while multiple infections between helminthes-helminthes species and helminthes-protozoa were $2.6 \%$.The highest co-infection rate was between $A$. lumbricoides and E. histolytica at $35.7 \%$. The most important environmental factors predisposing children to intestinal parasitic infections were drinking unsafe water and lack of self hygiene. The personal hygiene factors predisposing children to infection with intestinal parasites were failure to boil drinking water, failure to wash hands with soap after visiting toilets and failure to wash fruits before eating. Most of the agents identified in the study exhibited decreasing prevalence with increasing age.This study concluded that there was high level of intestinal parasitic infections among the primary school children with associated transmission risks. It is recommended that health education on personal hygiene and environmental sanitation be adopted in primary schools in order to reduce intestinal parasitic infections among children. The public health sector should provide health education on environmental sanitation and personal hygiene practices and promote environmental sanitation in primary schools.

\section{Competing interests}

The authors declare that they have no competing interests regarding the publication of this manuscript.

\section{Author's contributions}

MD and AA conceptualized the idea and drafted the initial proposal. AA was finalized the study proposal. MD and AA were leading the development of the data collection tools. AA contributed to data processing and analysis. MD drafted the manuscript. Both authors read and approved the manuscript.

\section{Acknowledgments}

The authors would like to thank Wollega University, College Natural and Computational Sciences for financial and logistics, Local Administration, School principals, Dembi Dolo hospital, laboratory technicians and study participants

\author{
Author details \\ ${ }^{1}$ Msc. In Biology, high school teacher, Kelem Secondary School, Dembi Dolo \\ ${ }^{2 *} \mathrm{PhD}$. in Biomedical Sciences, currently working as an Assistant professor under college of natural and \\ computational sciences, Department of Biology, Wollega University.
}

\section{References}

Abate, A., Kibret, B., Bekalu, E., Abera, S., Teklu, T., Yalew, A., Endris, M., Worku, L.and Tekesteet, Z. (2013). Cross-sectional study on prevalence of intestinal parasite and associated risk factors in Teda Health center 
northwest Ethiopia.International Scholarly Research Notices. Article ID 75451.

Alyousefi, N.A., Mahdy, M.A.K., Mahmud, R. and Lim, Y.A.L.(2011). Factors associated with high prevalence of intestinal protozoan infections among patients in Sana'a city Yemen.Public Library of Science 6: 1-7.

Ashford, R. W., and Crewe, W. (1998). The parasites of Homo sapiens. Cromwell Press, Liverpool.

Auta, T., Kogi, E. and Audu, O. (2013). Studies on the intestinal helminthes infestation among primary school children in Gwagwada, Kaduna, northwestern Nigeria. Journal of Biology, Agriculture and Healthcare 3:2224-3208.

Bayissa, C.(2013). Prevalence of intestinal parasitic infection in Mojo health center eastern Ethiopia: A6-year (2005-2010) retrospective study. Epidemiology 3:1.

Crompton, D. W. (1999). How much human helminthiasis is there in the world? Journal of Parasitology 85:397403.

El-Masry, H.M., Ahmed, Y.A., Hassan, A.A., Zaky, S. and Abd- Allah, E.S. (2007). Prevalence, risk factors and impacts of schistosomal and intestinal parasitic infections among rural school children in Sohag Governorate. Egypt Journalof Hospital Medicine 29: 616-630.

Emmy-Egbi, I.O. (2012). Prevalence of Intestinal helminthes in students of Ihiala local government area of Anambra state. Journal of Applied Technology and Environmental Sscience 2: 23-30.

FMHACA- Food, Medicine and Healthcare Administration and Control Authority of Ethiopia (2014). Standard treatment guidance for general hospital, 3rd edition.

Harhay, M.O., Horton, J. and Olliaro, P.L. (2010). Epidemiology and control of human gastrointestinal parasites in children. Expert Review Anti Infection 8:219-234.

Heckendorn, F., N'goran, E.K. and Felger, I. (2002).Species-specific field testing of Entamoeba species in an area of high endemicity. Transactions of Royal Society of Tropical Medicine and Hygiene 96:521-528.

Kirby, a., Gebski, V. and Keec, A.C. (2002). Determining the sample size in a clinical trial Gelantology.Blackwel. Oxford. 177:256-7.

Petri, W.A., Jr., Haque, R., Lyerly, D. and Vines, R.R.(2000). Estimating the impact of amebiasis on health. ParasitologyToday 16:320-21.

Sah, R.B., Pokharel, P.K., Paudel, I.S., Acharya, A. and Jha, N.(2013). A study of prevalence of intestinal protozoan infections and associated risk factors among the school children of Dharan, Eastern Region of Nepal. Journal of Medical Science Research 4 (1):11-15.

Stephenson, L.S., Latham, M.C. and Ottesen, E.A. (2000). Malnutrition and parasitic helminthes infections. Society of Medicie 13:1-15.

Tadesse, G. (2005). The prevalence of intestinal helminthic infections and associated risk factors among school children in Babile town, Eastern Ethiopia. Ethiopian Journal of Health Development 19:140-7.

Teklehaymanot, T. (2009). Intestinal parasitosis among Kara and Kwego semipastoralis Tribes in lower Omo valley, Southwestern Ethiopia. Ethiopian Journal of Health Development 23:57-62.

Thiong, F.W., Luoba, A. and Ouma, J.H. (2009).Intestinal helminthes and protozoa infections among school children in a rural district in Western Kenya. Biomed Central Public Health 9:23-25.

Ukpai, O.M. and Ugwu, C.D. (2003). The prevalence of gastro-intestinal tract parasites primary school children in Ikwuano local government area of Abia State, Nigeria. Journal of Parasitology 24(1):129-136.

Wang, X., Zhang, L., Luo, R., Wang, G. and Cheng, Y. (2012). Soil transmitted helminthes and correlated risk factors in pre-school and school age children in rural South West China. Public Library of Science Neglected Tropical Diseases 7(9):1371.

WHO (2010). Joint monitoring program for water supply and sanitation; Estimates for use of improved drinking water sources. WHO press, Geneva. WHO United nations Children's Fund. 342:714-756.

WHO (2011). Guidelines for drinking water quality.4th edition,WHO press, Geneva. 789:213-365.

Ziegebaver, K., Speich, B., Mausezahld, D., Bose, R., Keiser, J. and Utzinger J. (2012). Effects of s anitation on soil transmitted infections; Systematic review and meta-analysis. Public Library of Science Medicine 9(1):1317-1385. 